\title{
Application of nuclear techniques in two-phase liquid-solid particles hydrotransport investigations
}

\author{
Marcin Zych ${ }^{1, a}$, Robert Hanus ${ }^{2}$, Pavel Vlasak ${ }^{3}$, Leszek Petryka ${ }^{1}$ and Marek Jaszczur ${ }^{1}$ \\ ${ }^{1}$ A GH University of Science and Technology, Krakow 30-059, Poland \\ ${ }^{2}$ Rzeszow University of Technology, Rzeszow 35-959, Poland \\ ${ }^{3}$ Institute of Hydrodynamics AS CR, v. v. i., 16000 Prague, Czech Republic
}

\begin{abstract}
The paper presents gamma radiation application to two-phase flow investigation in a vertical pipeline, where the flow of solid particles transported by water was examined by use of both: radiotracers and gamma-absorption method. The simultaneous use of two methods allows analyzing of important parameters of solid particles hydrotransport. In the described experiments as solid phase the ceramic models representing natural polymetallic ocean nodules were used. Radiotracers allow to track the movements of selected models, representing specified grain size and the designation of its velocity. However gamma-absorption method enables measurement of average solid-phase velocity. For analysis of electrical signals obtained from scintillation detectors the cross-correlation method has been applied.
\end{abstract}

\section{Introduction}

Two-phase liquid-solid particles flow occurs often in the mining industry. An example would be vertical pipeline hydrotransport of minerals, e.g. polymetallic nodules. Nodules are porous organic and mineral compositions which contain a various metals (mostly $\mathrm{Mn}, \mathrm{Si}, \mathrm{Fe}, \mathrm{Al}$, $\mathrm{Na}, \mathrm{Mg}, \mathrm{Ni}, \mathrm{K}, \mathrm{Cu}$ ) [1-4]. They occur on the bottom of seas and oceans, but the richest deposits can be found at the Pacific Ocean bed in depth about $5000 \mathrm{~m}$ in the so called Clarion-Clipperton zone. The ocean nodules usually have the form of irregular grains with the diameter of several $\mathrm{mm}$ up to $0.5 \mathrm{~m}$ and density of about $2 \mathrm{~g} / \mathrm{cm}^{3}$ in wet state. The mining of nodules using the hydraulic method requires nodules vertical transport by water to sea level in extremely hard and varying environment. Determination of the velocity of nodules of various sizes in the vibrating pipeline at a significant depth is a very difficult task and requires the use noninvasive measurement techniques $[5,6]$.

One of these techniques, which are employed for many years in measurements of two-phase flows in pipelines and open channels, is a method using radioactive isotopes. In nuclear techniques radiotracer is injected under certain conditions into the flow and/or sealed radioactive sources are used [7-19]. In both cases as detectors of radiation the scintillation probes are mounted outside of the analyzed stream.

Typically in such measurements the mutually delayed stochastic signals are provided by detectors located on the outer walls of the pipeline. Measured time delay of signals is applied to determine the velocity of the marked phase (radiotracers method) or averaged velocity of the solid phase (absorption method). In the carried out experiments solid phase has been modeled using ceramic particles representing three grain sizes of natural polymetallic ocean nodules.

The processing of stochastic signals from scintillation probes requires the use of statistical methods in the time and frequency domain [20-26]. The cross-correlation function $(C C F)$ is the most known methods of time delay estimation applied for stationary random signals.

\section{Application of radioisotopes to investigations of nodules hydro- transport in a vertical pipeline}

The basic principle of gamma-ray absorption measurement is based on fact that the intensity of a collimated gamma beam decreases as it passes through matter [5-7, 10-17].

Radiotracer idea is based on marked particle tracking using a scintillation probes placed along the pipeline $[7$, $8,18]$

The principle of both methods and typical gammaradiation measuring equipment for liquid-solid particles flow evaluation in a vertical pipeline is presented in figure 1.

\footnotetext{
a Corresponding author: zych@geol.agh.edu.pl
} 


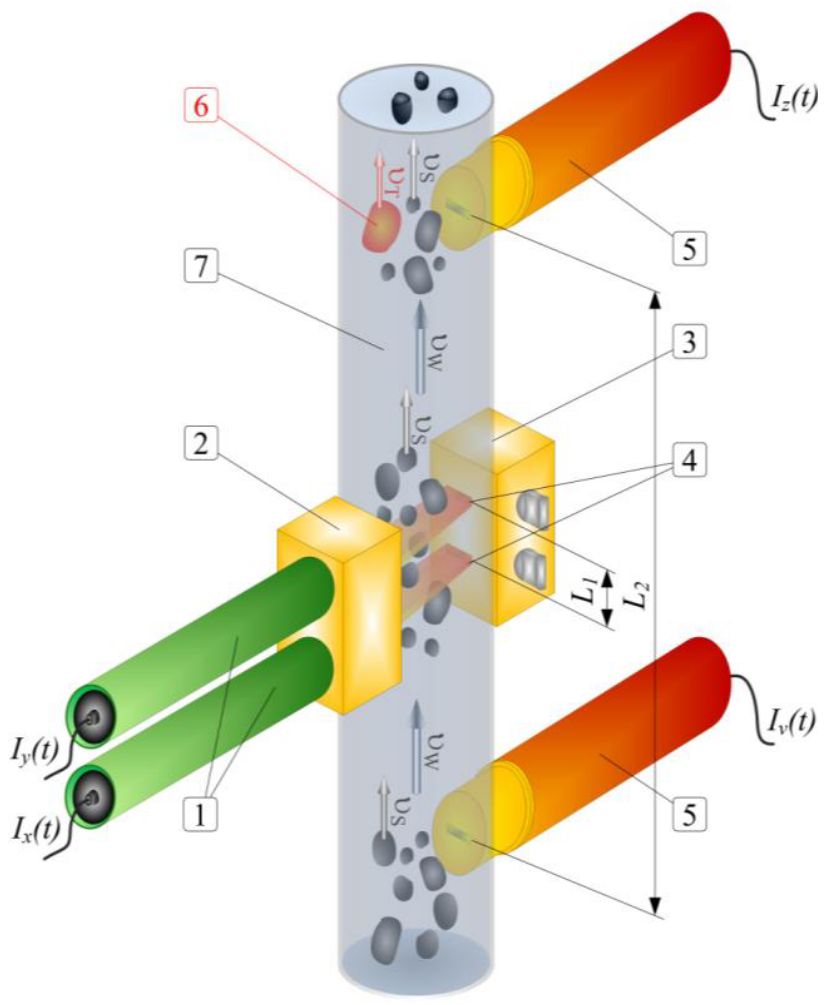

Figure 1. The gamma radiation measuring equipment: 1 - scintillation probes of gamma-absorption, 2 - detector's collimator, 3 - two gamma-radiation sources with collimator, 4 - gamma-rays, 5 - scintillation probe for radiotracer measurements, 6 - marked particle, 7 - pipeline with two-phase flow, $v_{S}, v_{W}$ - velocities of solid particles and water respectively, $v_{T}-$ velocity of marked particles.

Two sealed radioactive sources in collimator (3) emit gamma radiation beams shaped by the collimator. Photons pass through the pipeline (7) with the multiphase mixture. The changes in the intensity of radiation are recorded by the scintillation probes of the absorption set (1) with collimators (2) and next converted into output electrical impulses [7]. Count rates $I_{x}$ and $I_{y}$ at the outputs of probes depend on the flowing medium content in the test cross section. In the present investigations a linear ${ }^{241} \mathrm{Am} \gamma$-ray sources with an activity of $100 \mathrm{mCi}$ and probes with 2 " $\mathrm{NaI}(\mathrm{Tl})$ scintillation crystal were used. Two such sets were mounted on the pipe with the distance of $L_{l}=90 \mathrm{~mm}$ between them. Based on the time delay of signals $I_{x}$ and $I_{y}$ and the distance $L_{l}$ one can calculate the average velocity of the solid phase.

Probes for radiotracer measurements (5) are based on the longer distance of $L_{2}=1882 \mathrm{~mm}$. These probes record the radiation intensity $I_{v}$ and $I_{z}$ from the marked particle (6). Velocity of the marked particle can be determined as before by determining time delay between signals.

The current study has been performed for a mixture of water and artificial nodules at volumetric concentration 0.05 . The density of the particles representing the nodules was $2000 \mathrm{~kg} / \mathrm{m}^{3}$.

Table 1 presents the characteristics of solid particles and figure 2 shows image of particles before experiments.
Table 1. Characteristics of the artificial nodules.

\begin{tabular}{|c|c|c|}
\hline $\begin{array}{c}\text { Particle size } \\
\text { grade }\end{array}$ & $\begin{array}{c}\text { Dimensions } \\
(\mathrm{mm})\end{array}$ & $\begin{array}{c}\text { Weight, } \\
(\mathrm{g})\end{array}$ \\
\hline I - small & $29.5 \times 26.9 \times 20.5$ & 18.5 \\
\hline II - medium & $31.2 \times 47.7 \times 27.0$ & 47.9 \\
\hline III - big & $59.6 \times 43.7 \times 54.1$ & 128.3 \\
\hline
\end{tabular}

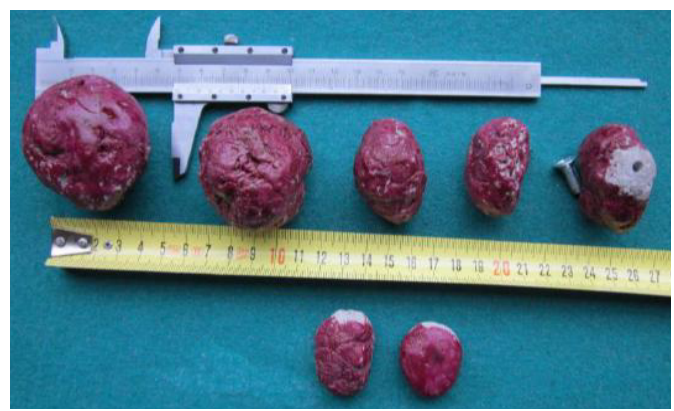

Figure 2. The image of the particles (right-hand side: the particle with a bore for the isotope Tc-99m).

\section{Experimental set-up}

The measuring system presented in figure 1 has been installed in the experimental set-up located in the Water Laboratory of the Wroclaw University of Environmental and Life Sciences, Poland. The entire experimental installation presented in figure 3 has been used for investigation of the polymetallic nodules hydrotransport.

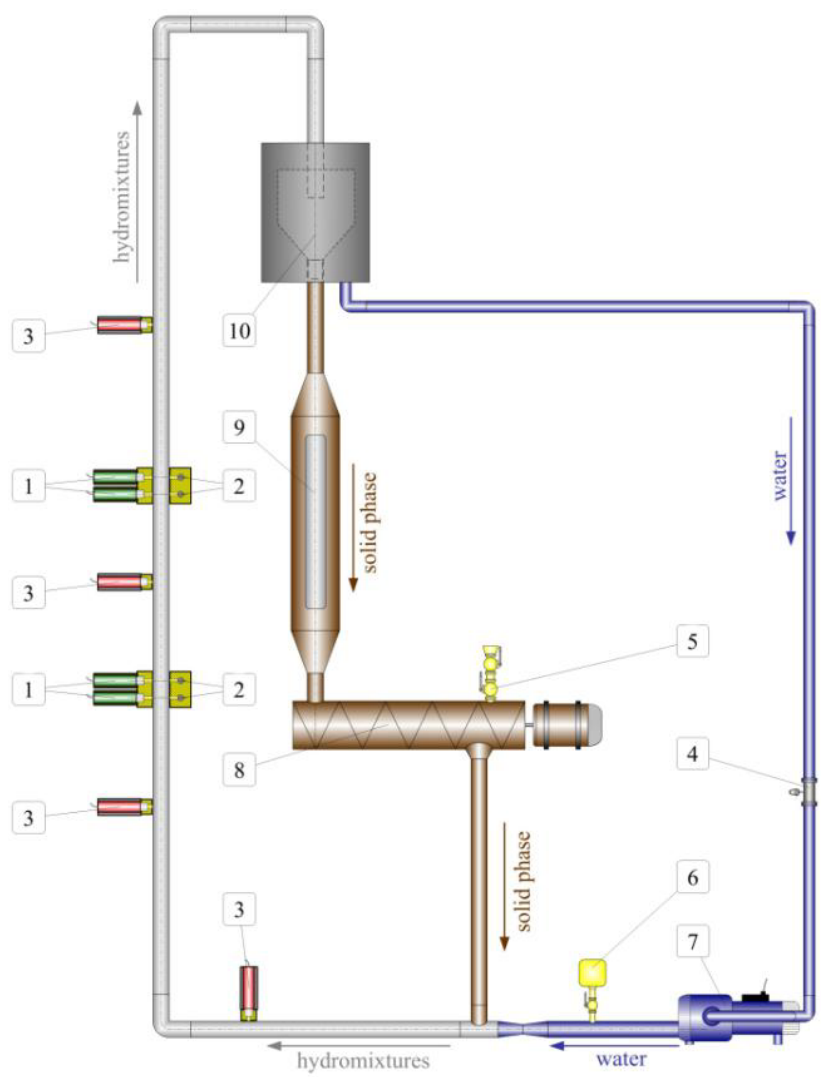

Figure 3. Diagram of experimental set-up: 1 - absorption probes, 2 - sealed sources, 3 - probes recording radiotracers, 4 - electromagnetic flow meter, 5 - marked particles feeder, 6 - feeder of marked water, 7 - pump, 8 - conveyer, 9 - container for solid particles, 10 - separating container. 
The key part of the installation is a vertical pipeline of length $7.75 \mathrm{~m}$ and with an internal diameter of $150 \mathrm{~mm}$ made of acrylic glass. The laboratory set-up has been described in details in papers $[5,13,16]$. The view of some parts of the experimental installation together with measuring section and gamma-absorption set as well as probe for radiotracer experiments is shown in figure 4 .

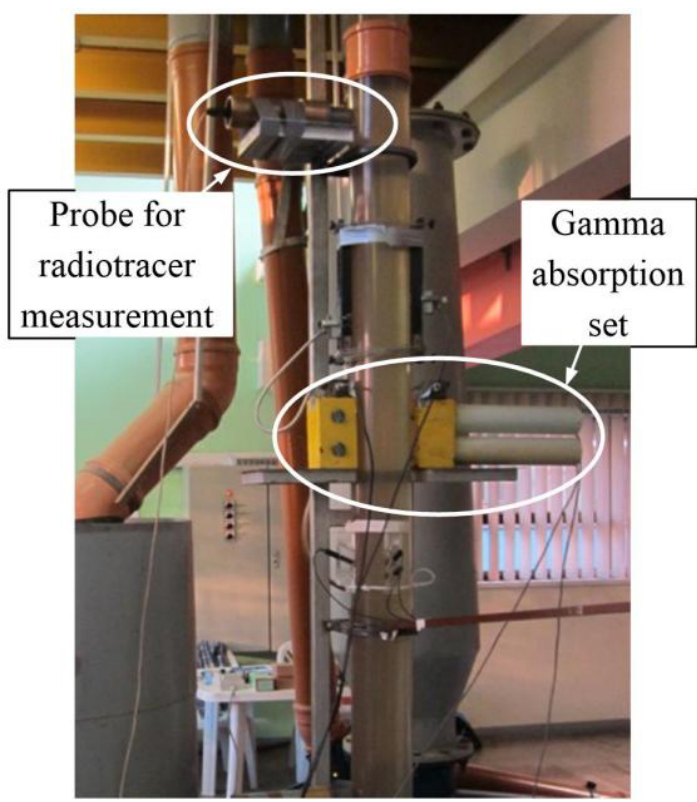

Figure 4. Test section view of the experimental set-up.

The data acquisition equipment was comprised of the dedicated 8-channel EC Electronics counter of HSC 8000 connected to $\mathrm{PC}$ using a USB port.

\section{The analysis of signals}

Signal pulses $I_{x}, I_{y}, I_{v}$ and $I_{z}$ from the four probes are counted within the selected sampling time $\Delta t=1 \mathrm{~ms}$ and create mutually delayed discrete stochastic signals $x(t)$, $y(t), v(t)$ and $z(t)$.

An examples of the time records $v(t)$ and $z(t)$ obtained in the WRS0057 experiment (particle size grade I) are presented in figure 5 , where s1, s2, s3, s4 denote consecutive marked particle passes through a measuring section of the pipe.

For comparison, the signals $x(t)$ and $y(t)$ from the probes of absorption set recorded at the same experiment is shown in figure 6.

The signals recorded at two spatially separated location within the distance $L_{l}$ allow determination of the $\tau_{0 S}$ time delay (transit time) necessary for transportation of the solid particles through the measuring section of the pipe. The particles average velocity $v_{S}$ can be calculated from the following formula:

$$
v_{S}=L_{1} / \tau_{0 S}
$$

The velocity of the marked particles $v_{T}$ is calculated as follows:

$$
v_{T}=L_{2} / \tau_{0 T}
$$
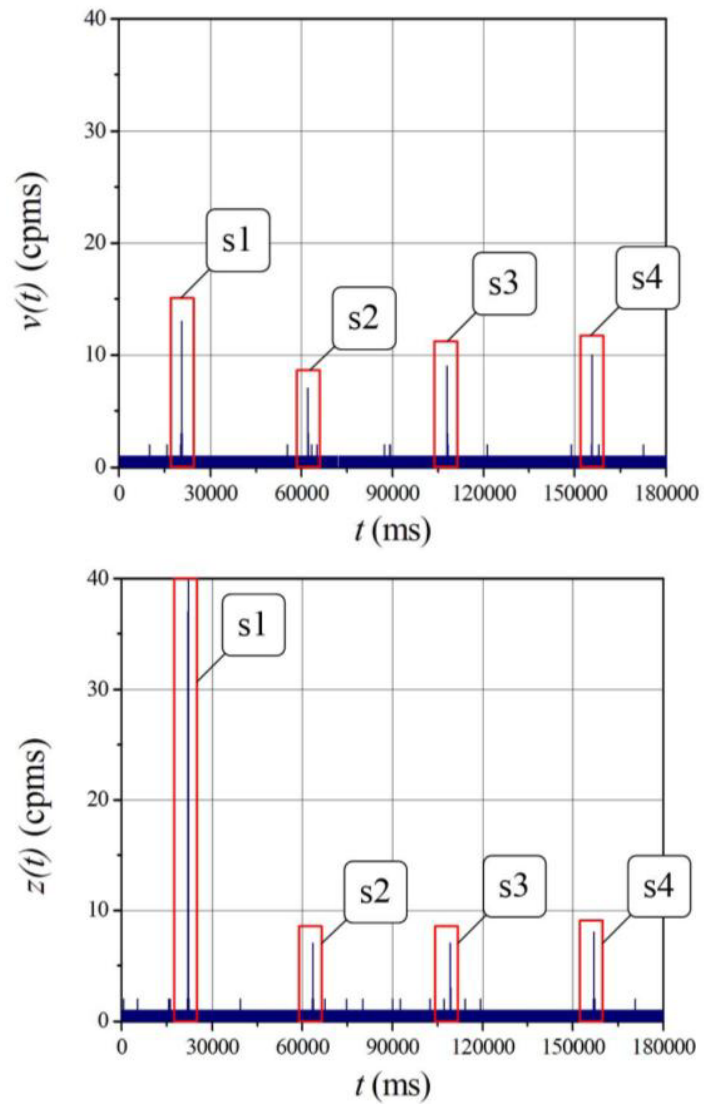

Figure 5. Time records of signal $v(t)$ and $z(t)$ obtained in the WRS0057 experiment.
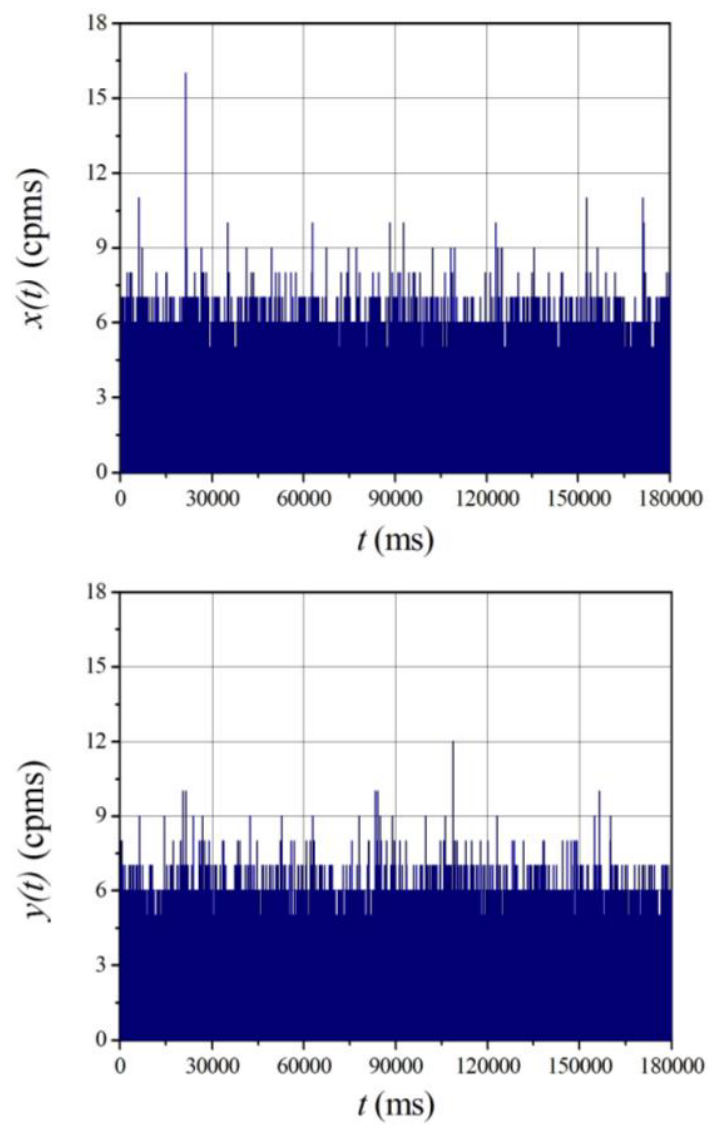

Figure 6. Time records of signal $x(t)$ and $y(t)$ in obtained from absorption set in the WRS0057 experiment 
The frequently used method of time delay estimation of the ergodic random signals $x(t)$ and $y(t)$ is based on the cross-correlation function $(C C F) R_{x y}(\tau)$, defined by equation [20-24]:

$$
R_{x y}(\tau)=\lim _{T \rightarrow \infty} \frac{1}{T} \int_{0}^{T} x(t) \cdot y(t+\tau) d t
$$

where $T$ is the averaging time, $\tau$ - time delay.

The transportation time delay $\tau_{0}$ is determined base on position of the $C C F$ global maximum.

\section{Results}

The graphs of the cross-correlation functions obtained in the WRS0057 experiment are shown in figure 7. Figure 7a presents $C C F$ for $v(t)$ and $z(t)$ signals obtained for marked particle and figure $7 \mathrm{~b}$ presents $C C F$ for signals from absorption set.

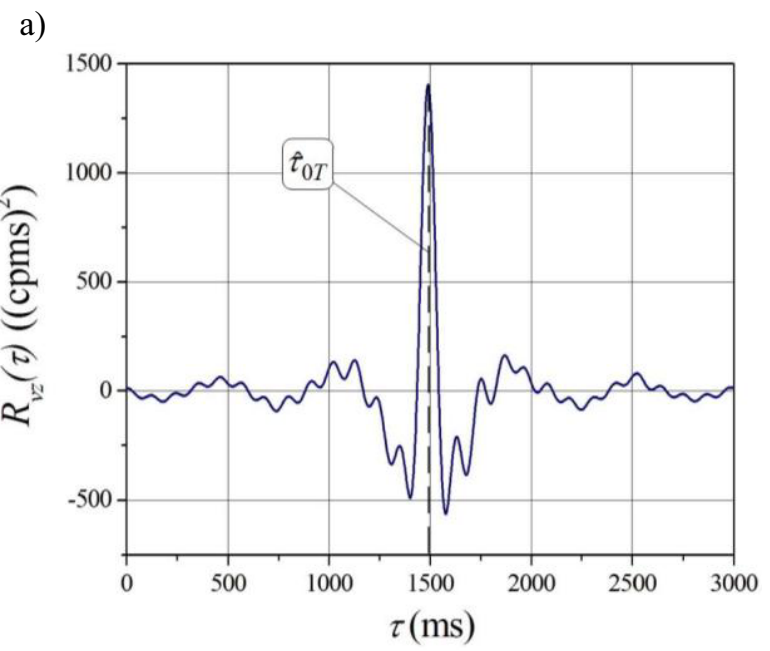

b)

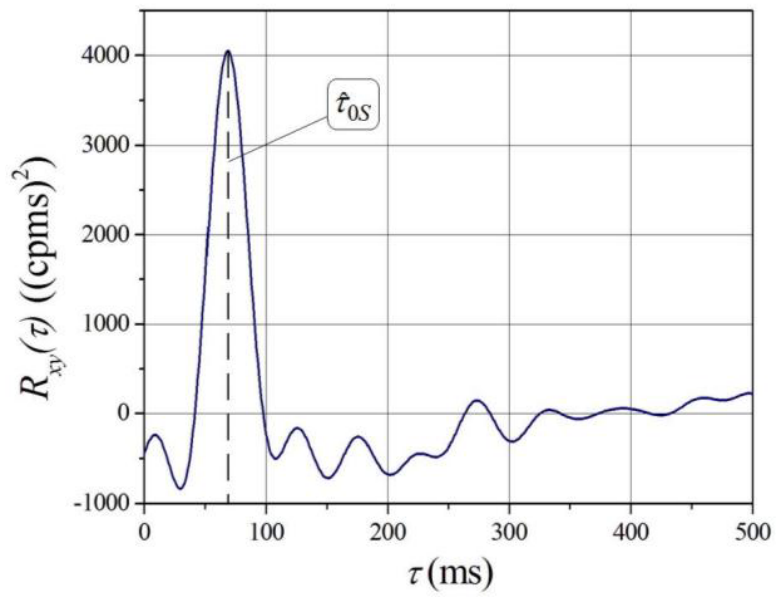

Figure 7. $C C F$ calculated in the WRS0057 experiment; (a) for signals from radiotracers (b) for signals from absorption set.

For correct interpretation of absorption and radiotracers experiments simultaneously conducted for different distances $L_{1}$ and $L_{2}$, the CCFS were converted to the function of inverse velocity. The converted $C C F s$, which include amplitude adjustment, are shown in figure 8 .
The combined standard uncertainty $u_{c}(v)$ of the solids velocity, with negligible small uncertainties of the acquisition set, depends on an inaccuracy of uncorrelated $L$ and $\tau_{0}$ determination [27]:

$$
u_{c}(v)=\left[\left(\frac{\partial v}{\partial L}\right)^{2} \cdot u^{2}(L)+\left(\frac{\partial v}{\partial \tau_{0}}\right)^{2} \cdot u^{2}\left(\tau_{0}\right)\right]^{1 / 2}
$$

where $u(L)$ is the standard uncertainty of the distance between detectors.

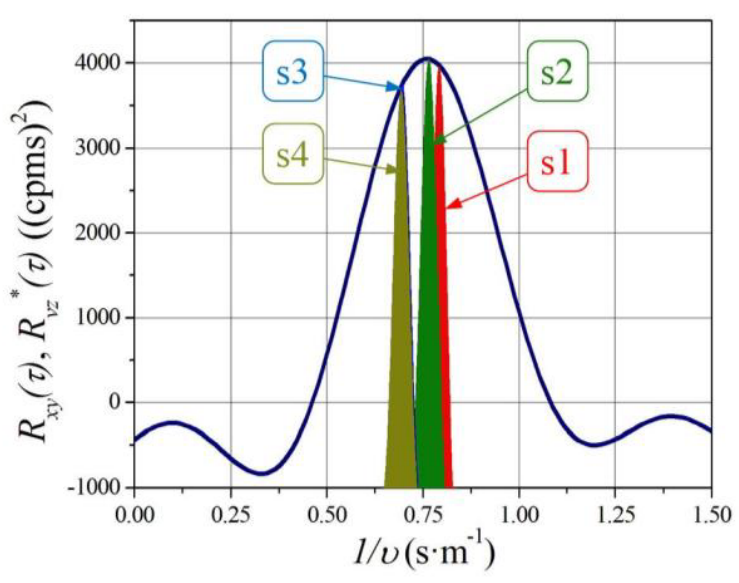

Figure 8. $C C F$ for signals obtained for marked particles (colored areas) and for signals from absorption set (solid line) in the WRS0057 experiments.

Table 2 shows the results of the marked solid particle velocity $v_{T}$ obtained from radiotracer measurements and its uncertainty. The average particle velocity $v_{S}$ calculated using absorption method is $v_{S}=1.323 \mathrm{~m} / \mathrm{s}$ with uncertainty $u_{c}\left(v_{S}\right)=0.031 \mathrm{~m} / \mathrm{s}$.

Table 2. Results of the marked solid particle velocity measurement in the WRS0057 experiment.

\begin{tabular}{|c|c|c|}
\hline Type of experiment & $\boldsymbol{v}_{\boldsymbol{T}}(\mathbf{m} / \mathbf{s})$ & $\boldsymbol{u}_{\boldsymbol{c}}\left(\boldsymbol{v}_{\boldsymbol{T}}\right)(\mathbf{m} / \mathbf{s})$ \\
\hline tracer, s1 & 1.2632 & 0.0023 \\
\hline tracer, s2 & 1.3078 & 0.0026 \\
\hline tracer, s3 & 1.4373 & 0.0030 \\
\hline tracer, s4 & 1.4435 & 0.0013 \\
\hline average & 1.3630 & 0.0048 \\
\hline
\end{tabular}

From the analysis of figure 8 and Table 2 it can be seen that velocity of the marked particles are in the range of greater $v_{S}$ values of the CCF distribution determined for absorption measurement. Velocities for s1, s2, s3, s4 are arranged within $\pm 1 \sigma$ (where $\sigma$ is standard deviation) aforementioned $C C F$.

The tracer experiment gives the ability to track individual selected grains whose velocity depends on the position in the flow. In the case of large number of radiotracer experiments when the number of passes indicated in the installation of stones is over 50, the calculated velocity values should be close to the $C C F$ distribution obtained from absorption measurements. 


\section{Conclusions}

Radiotracer experiments allow tracking the behavior of the selected grain size classes of the solid phase in the liquid-solids particle flow in vertical pipeline. This is important for the development of technology of hydrotransport of polymetallic nodules from the seabed.

The absorption measurements give an average velocity of the entire population of solid particles. The resulting distribution of the $C C F$ can be considered as distribution of all possible time delays (velocities) of the moving grain population.

Comparison of the results of both methods indicates that the velocities of marked particles obtained for s1, s2, $\mathrm{s} 3, \mathrm{~s} 4$ are arranged within $\pm 1 \sigma$ aforementioned $C C F$.

Measurements of radiotracers, due to the use of open radioisotopes must be performed under conditions of radiological protection. However, in many cases of flow measurements there is no alternative method that gives similar opportunities.

\section{Acknowledgment}

The authors would like to thank team of prof. Jerzy Sobota for their cooperation during the measurements undertaken at Wroclaw University of Environmental and Life Sciences.

All presented investigations were possible due to support under the project P105/10/1574 of the Grant Agency of the Czech Republic, and RVO: 67985874 of the ASCR, as well as the Project No NN523755340 of the National Science Centre of Poland, which are gratefully acknowledged.

This publication is funded by AGH University of Science and Technology (No 11.11.140.645).

\section{References}

1. D.S. Cronan (ed.), Handbook of marine mineral deposits (CRC Press, Boca Raton, 2000)

2. R. Kotliński, http://www.iom.gov.pl/uploads/iom attachment-95635.pdf

3. S. Depowski, et al, Mineral resources of the seas and oceans (SCHOLAR, Warsaw, 1998)

4. http://www.iom.gov.pl/
5. L. Petryka, et al., EPJ WoC 92, 02065 (2015)

6. R. Hanus, et al., EPJ WoC 92, 02020 (2015)

7. G. Falcone, G.F. Hewitt, C. Alimonti, Multiphase flow metering: principles and applications (Elsevier, Amsterdam, 2009)

8. G.A. Johansen, P. Jackson, Radioisotope gauges for industrial process measurements (Wiley, New York, 2004)

9. M. Zych, et al., Flow Meas. Instrum. 35, 11-15 (2014)

10. Z. Yu, B. Qincheng, H. Richa, Appl. Therm. Eng. 60, 398-410 (2013)

11. M. Zych, et al., EPJ WoC 92, 02122 (2015)

12. W.A.S. Kumara, B.M. Halvorsen, M.C. Melaaen, Int. J. Multiphas. Flow 36, 467-480 (2010)

13. R. Hanus, L. Petryka, M. Zych, Flow Meas. Instrum. 40, 58-63 (2014)

14. M. Zych, et al., MATEC WoC 18, 02004 (2014)

15. B.K. Arvoh, R. Hoffmann, M. Halstensen, Flow Meas. Instrum. 23, 56-65 (2012)

16. M. Zych, et al., Prz. Elektrotech. 88 (10a), 98-100 (2012)

17. S.H. Jung, et al., Appl. Rad. Isotop. 67, 1254-1258 (2009)

18. H.J. Pant, et al., Appl. Radiat. Isotop. 67, 16091615 (2009)

19. P. Vlasak et al., J. Hydrol. Hydrom. 62, 241-247 (2014)

20. M.S. Beck, A. Plaskowski, Cross correlation flowmeters - their design and application. (Adam Hilger, Bristol, 1987)

21. R. Hanus, Int. J. Multiphas. Flow 72, 210-217 (2015)

22. J.S. Bendat, A.G. Piersol, Random data - analysis and measurement procedures (Wiley, New York, 2010)

23. R. Hanus, et al., Math. Probl. Eng. 2014, 475735 (2014)

24. S.L. Soo (ed.), Instrumentation for fluid-particle flow (Noyes Publications, New Jersey, 1999)

25. R. Hanus, M. Zych, L. Petryka, JPCS 530, 012042 (2014)

26. R. Hanus, Prz. Elektrotech. 86 (6), 232-235 (2010)

27. Guide to the expression of uncertainly in measurement (International Organisation for Standardisation, 1995) 